\title{
Förster Coupling in Nanoparticle Excitonic Circuits
}

\section{Citation}

Rebentrost, Patrick, Michael Stopa, and Alán Aspuru-Guzik. 2010. Förster coupling in nanoparticle excitonic circuits. Nano Letters 10(8): 2849-2856.

\section{Published Version}

doi: $10.1021 / \mathrm{nl} 1008647$

\section{Permanent link}

http://nrs.harvard.edu/urn-3:HUL.InstRepos:8347338

\section{Terms of Use}

This article was downloaded from Harvard University's DASH repository, and is made available under the terms and conditions applicable to Open Access Policy Articles, as set forth at http:// nrs.harvard.edu/urn-3:HUL.InstRepos:dash.current.terms-of-use\#OAP

\section{Share Your Story}

The Harvard community has made this article openly available.

Please share how this access benefits you. Submit a story.

\section{Accessibility}




\title{
Förster coupling in nanoparticle excitonic circuits
}

\author{
Patrick Rebentrost, ${ }^{1}$ Michael Stopa,${ }^{2}$ and Alán Aspuru-Guzik ${ }^{1}$ \\ ${ }^{1}$ Department of Chemistry and Chemical Biology, Harvard University, 12 Oxford St., Cambridge, MA 02138 \\ ${ }^{2}$ Center for Nanoscale Systems, Harvard University, 11 Oxford St., Cambridge, MA 02138
}

(Dated: July 6, 2010)

\begin{abstract}
Exciton transport in semiconductor nanoparticles underlies recent experiments on electrically controlled excitonic circuits and proposals for new artificial light-harvesting systems. In this work, we develop a new method for the numerical evaluation of the Förster matrix element, based on a three-dimensional real space grid and the self-consistent solution of the mesoscopic exciton in a macroscopic dielectric environment. This method enables the study of the role of the nanoparticle shape, spatially varying dielectric environment, and externally applied electric fields. Depending on the orientation of the transition dipole, the Förster coupling is shown to be either increased or decreased as a function of the nanoparticle shape and of the properties of the dielectric environment. In the presence of an electric field, we investigate the relation between excitonic binding and confinement effects. We also study a type II core-shell quantum dot where electron and hole are spatially separated due to a particular configuration of the bandstructure.
\end{abstract}

PACS numbers: PACS number

When a photon produces an electron-hole pair, or exciton, localized on a molecule, quantum dot or nanoparticle site, the excitation energy can transfer from this donor site to a neighboring acceptor site via coupling of the electrons to the electromagnetic field. This process is known as fluorescent resonant energy transfer (FRET) [1]. FRET, or, more generally, the matrix element linking together donor and acceptor transitions, underlies energy transport between chlorophyll molecules in photosynthesis [1,2], and is essential for various existing and envisioned nano-structured devices such as artificial light-harvesting systems [3-5] and quantum computation implementations [6, 7]. Thus, in addition to the desire to understand FRET in naturally occurring systems, the many photonic engineering systems currently under investigation motivate studies aimed at controlling the excitons and their interactions. As one example, tunability of the energy transfer between a nanorod and a dye molecule by an electric field was shown in [8]. In another case, control of exciton diffusion in excitonic integrated circuits was recently displayed [9].

Förster theory [10] describes exciton transfer based on Fermi's golden rule and involving three main quantities: the spectral overlap between donor emission and acceptor absorption, a dielectric screening factor, and the coupling matrix element due to Coulombic interactions. The spectral overlap for quantum dots was experimentally studied in [5, 11], for example. Theoretically, the absorption and emission spectra can be computed using the energy levels of donor and acceptor and the line shape function. The latter is dependent on the spectral density which describes the coupling to phonons [12]. We focus on dielectric effects and the matrix element. The matrix element has the form of a dipole-dipole coupling when obtained from an expansion of the bare Coulomb kernel in $a / R$, where $a$ is the radius of the sites, be they atoms, molecules or nanoparticles, and $R$ is the separation of the site centers. Theories beyond the Förster treatment include using a quantized treatment of the electromagnetic field [13, 14]. This method has the virtue of describing both the near field (or "radiationless" regime) and the far field ("radiation" regime) and their dependence on distance. A different direction of going beyond the Förster model involves the inclusion of the complex environment of the molecules or nanoparticles. Central to actual and proposed nanoscale devices are inhomogeneous dielectric materials and metal surfaces at externally controllable potentials. For these purposes, the Coulomb kernel becomes more complicated than the simple vacuum interaction and a more refined treatment than an expansion in $a / R$ becomes necessary. Such a realistic treatment of the environment is one principal objective of this work.

Distinct from the form of the interaction in FRET, what we may call the electromagnetic part of the problem, is the nature of the excitonic states and what approximations are used to compute them. Many calculations exist in the literature for excitons in condensed matter and in particular lower dimensional systems. In the simplest case, the background is translationally invariant and the problem can be solved with two effective mass bands in center-of-mass coordinates [17]. For a multi-band, inhomogeneous systems, care must be taken to conserve the current of the full (Bloch plus envelope) wave function at the material interfaces [18]. For strong confinement, one can sometimes ignore the electron-hole Coulomb interaction altogether (or treat it as a perturbation) [16, 19]. Additionally, atomistic calculations at varying degrees of precision, have been carried out. For example, empirical tight binding calculations employing linear combination of atomic orbitals (LCAO) are able to include large numbers of atoms in studies of InAs quantum dots [15, 20]. These calculations capture strain effects, for example. However, such tight-binding studies employ a fundamentally single electron formalism and therefore cannot include excitonic binding or Coulomb correlation. Ab initio density functional theory calculations have also been performed for isolated nanocrystals (using numerical codes like VASP [21], for instance). These calculations have investigated the validity of the effective mass approximation [22]. A similar approach was used to study the effect of the shape of the nanoparticles on electronic structure [23] and, using the transition density cube method [24], the Förster coupling [25]. Nevertheless, ab initio calculations, are limited 
to a few hundred atoms at most and therefore do not typically capture the effect of the macroscopic-scale environment.

In this paper, we present our method for calculating the Förster coupling between nanoparticles of arbitrary geometry, i.e. size, shape, and orientation, embedded in an arbitrarily complex electrostatic environment of position-dependent dielectric constant $\epsilon(\mathbf{r})$ and metallic gates. The central theoretical advancement is an illustrative expression for the Förster coupling based on the gradients of the electron-hole transition densities and the fully screened Coulomb kernel. We calculate the electronic structure of an electron-hole pair in a nanoparticle employing single conduction band and single valence band effective masses. We self-consistently solve the SchrödingerPoisson equations on a real-space, three-dimensional mesh, include the excitonic binding energy, and employ a technique [26] for avoiding electron or hole self-interaction. We express the exciton envelope wave function as a single product of electron and hole wavefunctions. Correlation is thereby included by the self-consistency, but at an approximate level. A similar calculation for the bandgap renormalization in semiconductor quantum wires has been done and has shown good agreement with experimentally obtained exciton binding energies $[26,27]$.

The Bloch portions of the wave functions are taken as the bulk values of a single electron and single hole band. Thus, the orientation of the permanent transition dipole moment, which proceeds from the symmetry of the hole wave function [28] and which we will show appears prominently in the theory, is understood to result from the orientation of the crystal axes [19]. We do not consider that, for the case of small nanoparticles, the vanishing of the wave function at the boundaries mixes different Bloch states. This can lead to dipole moments of the exciton which depend upon the shape of the nanoparticle. The envelope portion of the Förster matrix element is calculated with the fully screened Coulomb interaction in a realistic electrostatic environment. The technique for accomplishing this, which is a central result of this paper, makes use of the numerical solution of Poisson's equation [29]. With this technique, the explicit calculation and storage of the screened Poisson kernel, $F\left(\mathbf{r}_{1}, \mathbf{r}_{2}\right)$, is not required. Additionally, the computation of the Förster matrix element is not limited by the condition, from the dipole approximation, $R \gg a$ [16]. Rather, the interaction is expanded about the center of a mesh cell, which can be made arbitrarily small. Hence, the appropriate condition on the accuracy of our calculation is that $R \gg \Delta$, where $\Delta$ is the size of the mesh cell.

\section{EXCITON IN A NANOPARTICLE}

A typical geometry of two nanoparticles is shown in Fig. 1. The calculations are all fully three-dimensional. Conduction $V_{c}(\mathbf{r})$ and valence $V_{v}(\mathbf{r})$ band offsets are assumed throughout the structure, except for the metallic regions (i.e. gates as in the inset of Fig. 4) which are treated with Dirichlet boundary conditions imposed on the Poisson equation. The band offsets trap the electron and hole in the nanoparticles. We solve for the exciton in each of the nanoparticles separately. In the a)

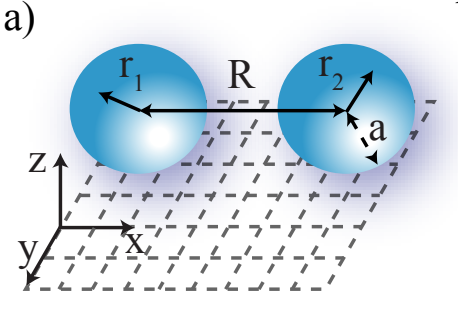

b)

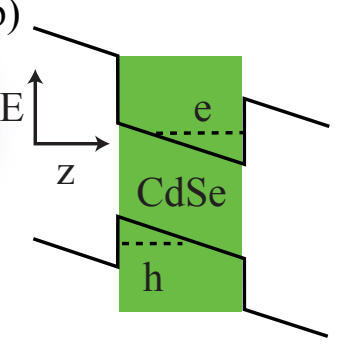

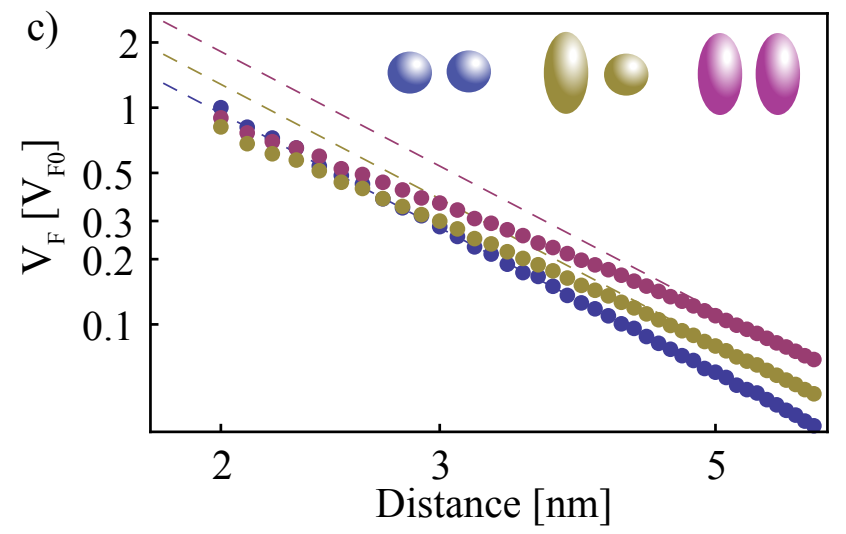

FIG. 1: (a) The general setup of this work is two nanoparticles that spatially confine an electron-hole pair or exciton and exhibit Förster coupling. The setup is simulated on a three-dimensional real-space mesh with arbitrary spatial dielectric function and metallic boundary conditions. The center-to-center distance is given by $\mathbf{R}$ and $a$ is the radius of the spherical nanoparticles (quantum dots). The vectors $\mathbf{r}_{1}$ and $\mathbf{r}_{2}$ point to an arbitrary unit cell within the respective particle. (b) Band structure in $z$ direction of a single quantum dot when an electric field is applied. In this work, the material for the nanoparticles is CdSe. (c) Distance dependence of the Förster coupling for two nanoparticles for different particle shapes, as depicted in the inset. The nanoparticle diameter is taken to be $2 \mathrm{~nm}$ and, for the non-spherical cases, the elongation is $5 \mathrm{~nm}$ perpendicular to the separation vector. The distance dependence is well described by a $1 / R^{3}$ dependence (dashed) for spherical nanoparticles, while for the elongated configurations a substantial deviation is obtained. For a distance of $2 \mathrm{~nm}$ (i.e. no spacing between the dots) the coupling is $\left|V_{F 0}\right|=2.72 \mathrm{meV}$ in the spherical case.

envelope function approximation the electron or hole wavefunction in the nanoparticle can be factorized as [30]:

$$
\Psi_{e / h}(\mathbf{r})=\psi_{e / h}(\mathbf{r}) U_{e / h}(\mathbf{r}),
$$

where $\psi_{e / h}(\mathbf{r})$ is the envelope wavefunction for electron or hole. It varies slowly on the length scale of the atomic lattice. The Bloch part of the wavefunction, $U(\mathbf{r})$, is periodic with the atomic lattice. The electron and hole envelope wavefunctions satisfy the coupled Schrödinger equations as follows:

$$
\begin{aligned}
{\left[\frac{-\hbar^{2}}{2 m_{e}^{*}} \nabla^{2}+V_{c}(\mathbf{r})+e \phi_{e}(\mathbf{r})\right] \psi_{e}(\mathbf{r}) } & =E_{e} \psi_{e}(\mathbf{r}), \\
{\left[\frac{-\hbar^{2}}{2 m_{h}^{*}} \nabla^{2}+V_{v}(\mathbf{r})+e \phi_{h}(\mathbf{r})\right] \psi_{h}(\mathbf{r}) } & =E_{h} \psi_{h}(\mathbf{r}),
\end{aligned}
$$


where the electron and hole effective masses are $m_{e}^{*}$ and $m_{h}^{*}$, respectively. The potentials $\phi_{e}$ and $\phi_{h}$ lead to the coupling of the two equations. Physically, the electrostatic potential for the electron is produced by the hole and by the image charges produced by both the hole and the electron. To obtain $\phi_{e}$, we begin with the total electrostatic potential defined by:

$$
-\nabla_{\mathbf{r}} \epsilon(\mathbf{r}) \nabla_{\mathbf{r}} \phi(\mathbf{r})=4 \pi\left(\rho_{e}(\mathbf{r})+\rho_{h}(\mathbf{r})\right),
$$

where $\rho_{e}(\mathbf{r}) \equiv\left|\psi_{e}(\mathbf{r})\right|^{2}$ and $\rho_{h}(\mathbf{r}) \equiv\left|\psi_{h}(\mathbf{r})\right|^{2}$. In the presence of metallic gates, Eq. (3) satisfies the corresponding Dirichlet boundary conditions at the surfaces of the gates which are kept at constant potentials. The spatially varying dielectric function is given by $\epsilon(\mathbf{r})$. The electron potential is then given as:

$$
\phi_{e}(\mathbf{r})=\phi(\mathbf{r})-\phi_{e}^{0}(\mathbf{r}),
$$

where $\phi_{e}^{0}(\mathbf{r})$ is obtained by solving Poisson's equation with only the electron charge $\rho_{e}(\mathbf{r})$ as a source, with the same dielectric background, $\epsilon(\mathbf{r})$, but with no metal gates. The numerical calculation of $\phi_{e}^{0}(\mathbf{r})$ is performed on an expanded mesh to allow accurate imposition of $1 / r$ boundary conditions at large $r$. Equation (4) eliminates the self-interaction of the electron. A similar procedure is carried out for the hole electrostatic potential $\phi_{h}(\mathbf{r})$.

The solution of Eqs. (2) must be iterated with the solution for the potentials $\phi_{e}$ and $\phi_{h}$ until the density is stationary. The resulting product of electron and hole wavefunctions, including the Bloch part, defines the nanoparticle transition density that arises in the Förster matrix element:

$$
M(\mathbf{r}) \equiv \psi_{e}^{*}(\mathbf{r}) \psi_{h}(\mathbf{r}) U_{e}^{*}(\mathbf{r}) U_{h}(\mathbf{r})=M_{E}(\mathbf{r}) M_{B}(\mathbf{r}),
$$

where we have defined the envelope transition density $M_{E}(\mathbf{r}) \equiv \psi_{e}^{*}(\mathbf{r}) \psi_{h}(\mathbf{r})$ and the Bloch transition density $M_{B}(\mathbf{r}) \equiv U_{e}^{*}(\mathbf{r}) U_{h}(\mathbf{r})$. As discussed in the following section, the Förster coupling matrix element is defined as the direct Coulomb coupling of two transition densities: one associated with the donor nanoparticle and one associated with the acceptor nanoparticle.

\section{FÖRSTER COUPLING OF NANOPARTICLES}

In this section, we develop our method for computing the Förster coupling matrix element. In the presence of a variable dielectric environment and electrostatic boundary conditions the Förster coupling matrix element is generally of the form:

$$
V_{F}=-\frac{e^{2}}{4 \pi} \iint d \mathbf{r}_{1} d \mathbf{r}_{2} M_{1}\left(\mathbf{r}_{1}\right) F\left(\mathbf{r}_{1}, \mathbf{r}_{2}\right) M_{2}^{*}\left(\mathbf{r}_{2}\right) .
$$

The transition densities of the two nanoparticles are given by $M_{1}$ and $M_{2}$. The Green's function $F\left(\mathbf{r}_{1}, \mathbf{r}_{2}\right)$ is the solution to:

$$
\nabla_{\mathbf{r}_{1}}\left(\epsilon\left(\mathbf{r}_{1}\right) \nabla_{\mathbf{r}_{1}} F\left(\mathbf{r}_{1}, \mathbf{r}_{2}\right)\right)=-4 \pi \delta\left(\mathbf{r}_{1}-\mathbf{r}_{2}\right),
$$

including again the appropriate boundary conditions.
First, for comparison, we give the dipole-dipole expression derived in Nazir et al. [7], where the interaction is taken to be of the translationally invariant form $F\left(\mathbf{r}_{1}, \mathbf{r}_{2}\right)=$ $1 / \epsilon_{0} \epsilon_{r}\left|\mathbf{R}-\mathbf{r}_{1}-\mathbf{r}_{2}\right|$. The separation vector $\mathbf{R}$ is defined between the centers of the two nanoparticles and the coordinates $\mathbf{r}_{1}$ and $\mathbf{r}_{2}$ are defined from the center of the nanoparticle, see Fig. 1 (a). A homogeneous dielectric background with relative dielectric constant $\epsilon_{r}$ is assumed. Because the Coulomb interaction is given in its free-space form, it is straightforward to expand the interaction in powers of $a / R$, where $a$ is the radius of the nanoparticle. This leads in lowest order to an interaction between the dipole terms in the charge distribution of the two nanoparticles [7]:

$$
V_{F}^{d-d}=\frac{-e^{2}}{4 \pi \epsilon_{0} \epsilon_{r}} \frac{O_{1} O_{2}}{R^{3}}\left(\mathbf{d}_{1} \cdot \mathbf{d}_{2}-\frac{3}{R^{2}}\left(\mathbf{d}_{1} \cdot \mathbf{R}\right)\left(\mathbf{d}_{2} \cdot \mathbf{R}\right)\right) .
$$

The characteristic $1 / R^{3}$ dependence of a dipole-dipole interaction is obtained. For this expression, one defines the overlap integrals:

$$
O=\int d \mathbf{r} M_{E}(\mathbf{r}),
$$

involving the envelope wavefunctions. The permanent material dipoles,

$$
\mathbf{d}=\int_{\Sigma} d \mathbf{r} M_{B}(\mathbf{r}) \mathbf{r}
$$

involve the Bloch wavefunctions and the integral is over a unit cell $\Sigma$. The dipoles $\mathbf{d}$ are material parameters which can be obtained from experiment or detailed atomistic calculations.

We now develop our generalized expression that goes beyond the point dipole approximation and incorporates dielectric effects, using $F\left(\mathbf{r}_{1}, \mathbf{r}_{2}\right)$ as the solution to Eq. (7). One can separate the coordinate $\mathbf{r}_{1}$ into a vector pointing to the center of a unit cell $\mathbf{R}_{1}$ and a vector within this unit cell $\mathbf{r}_{1}^{\prime}$, i.e. $\mathbf{r}_{1}=\mathbf{R}_{1}+\mathbf{r}_{1}^{\prime}$ (and similarly for $\mathbf{r}_{2}$ ). This allows us to Taylor-expand the Green's function around the unit cell center, i.e. $F\left(\mathbf{R}_{1}+\mathbf{r}_{1}^{\prime}, \mathbf{R}_{2}+\mathbf{r}_{2}^{\prime}\right)=F^{(0)}+F^{(1)}+F^{(2)}+\ldots$ . In the evaluation of the Förster matrix element the zeroth and first order contributions, $F^{(0)}$ and $F^{(1)}$ respectively, vanish because of the orthogonality of the Bloch wavefunctions [7]. The first non-vanishing term is the second-order term corresponding to $F^{(2)}=\frac{1}{2} \sum_{i, j} \mathbf{r}_{1, i}^{\prime} \mathbf{r}_{2, j}^{\prime} \partial_{\mathbf{R}_{1, i}} \partial_{\mathbf{R}_{2, j}} F\left(\mathbf{R}_{1}, \mathbf{R}_{2}\right)$, where the indices $i$ and $j$ are over the Carthesian coordinates. The calculation can be simplified by considering the different length scales of the nanoparticle and the unit cells and by separating the integration as $\int d \mathbf{r}_{1}=\sum_{\mathbf{R}_{1}} V_{\Sigma} \int_{\Sigma\left(\mathbf{R}_{1}\right)} d \mathbf{r}_{1}^{\prime}$, where $V_{\Sigma}$ is the volume of the unit cell [6]. The sum is over all lattice vectors and the integration is over the respective unit cell. The same relation holds for the coordinate $\mathbf{r}_{2}$. Using the periodicity of the Bloch function $U\left(\mathbf{R}_{1}+\mathbf{r}_{1}^{\prime}\right)=U\left(\mathbf{r}_{1}^{\prime}\right)$, the Förster matrix element is given by:

$$
\begin{aligned}
V_{F}= & -\frac{1}{2} \frac{e^{2}}{4 \pi} \sum_{i, j} \mathbf{d}_{1 i} \mathbf{d}_{2 j} \sum_{\mathbf{R}_{1}} V_{\Sigma} \sum_{\mathbf{R}_{2}} V_{\Sigma} \\
& \times M_{E 1}\left(\mathbf{R}_{1}\right) \partial_{\mathbf{R}_{1 i}} \partial_{\mathbf{R}_{2 j}} F\left(\mathbf{R}_{1}, \mathbf{R}_{2}\right) M_{E 2}^{*}\left(\mathbf{R}_{2}\right),
\end{aligned}
$$


with the unit cell dipole moments $\mathbf{d}_{1 / 2}$, see Eq. (10). An expression similar to Eq. (11) has been derived by Govorov [16], who also expands the Coulomb kernel about the unit cells (as opposed to about the dot centers), albeit using the translationally invariant interaction. Here, we maintain a general form for $F\left(\mathbf{R}_{1}, \mathbf{R}_{2}\right)$. Approximating the sum over lattice vectors by an integration and performing an integration by parts the Förster coupling matrix element in the presence of a variable dielectric environment is:

$$
\begin{aligned}
V_{F}= & -\frac{1}{2} \frac{e^{2}}{4 \pi} \int d \mathbf{R}_{1} \int d \mathbf{R}_{2} \\
& {\left[\mathbf{d}_{1} \cdot \nabla_{\mathbf{R}_{1}} M_{E 1}\left(\mathbf{R}_{1}\right)\right] F\left(\mathbf{R}_{1}, \mathbf{R}_{2}\right)\left[\mathbf{d}_{2} \cdot \nabla_{\mathbf{R}_{2}} M_{E 2}^{*}\left(\mathbf{R}_{2}\right)\right] }
\end{aligned}
$$

which involves the gradient of the envelope transition densities of both nanoparticles. This can be interpreted as follows. The Förster coupling is the Coulomb interaction between the gradient of the electron-hole overlap in one nanoparticle with that in the other nanoparticle. The gradient terms arise because a field, rather than a constant potential, is needed to couple the $\mathrm{p}$ and $\mathrm{s}$ wave functions of valence and conduction band. In contrast to earlier work, the integration by parts leading to Eq. (12) explicitly exhibits the dipoles as the gradients of the electron-hole overlaps. Equation (12) is the central theoretical result of this paper. It is especially amenable to numerical treatment, as we now demonstrate.

Note that in Eq. (12), the kernel of Poisson's equation $F\left(\mathbf{R}_{1}, \mathbf{R}_{2}\right)$ is not simply a function of $\mathbf{R}_{1}-\mathbf{R}_{2}$. It is in general very complicated and even if it were calculated numerically for a specific geometry of gates and dielectrics, its mere storage would require an array with $N^{2}$ elements, where $N$ is the number of mesh points in the simulation. Since $N$ is typically $\sim 10^{6}$ the storage requirements alone are prohibitive. We can, however, calculate matrix elements like Eq. (12) without actually determining the explicit form of the interaction $F\left(\mathbf{R}_{1}, \mathbf{R}_{2}\right)$, as follows. First, the Poisson equation for $\nabla_{\mathbf{R}_{2}}\left(\epsilon\left(\mathbf{R}_{2}\right) \nabla_{\mathbf{R}_{2}} \Phi_{j}\left(\mathbf{R}_{2}\right)\right)=$ $-4 \pi \partial_{\mathbf{R}_{2 j}}\left(M_{E 2}\left(\mathbf{R}_{2}\right)\right)$ is solved in a finite difference method (again with the appropriate dielectric background and boundary conditions) to obtain the potential $\Phi_{j}\left(\mathbf{R}_{1}\right)=$ $\int d \mathbf{R}_{2} F\left(\mathbf{R}_{1}, \mathbf{R}_{2}\right) \partial_{\mathbf{R}_{2 j}}\left(M_{E 2}\left(\mathbf{R}_{2}\right)\right)$. Then, the numerical evaluation of the integral $\int d \mathbf{R}_{1} \partial_{\mathbf{R}_{1}}\left(M_{E 1}\left(\mathbf{R}_{1}\right)\right) \Phi_{j}\left(\mathbf{R}_{1}\right)$ obtains the coupling $V_{F}$.

\section{RESULTS}

Our method lends itself to the study of engineered nanodevices and quantum dot networks. In this section, we present examples consisting of two nanoparticles which can be thought of as building blocks in a larger array of nanoparticles. While the examples illustrate our theoretical result and predict the properties of nanoparticle interactions in realistic situations, we also expect our method to perform efficiently when simulating larger setups and complete device architectures. Our results are divided into two parts. In the first part, we compute the dependence of the Förster coupling of two nanoparticles on the distance between them, their shape, and

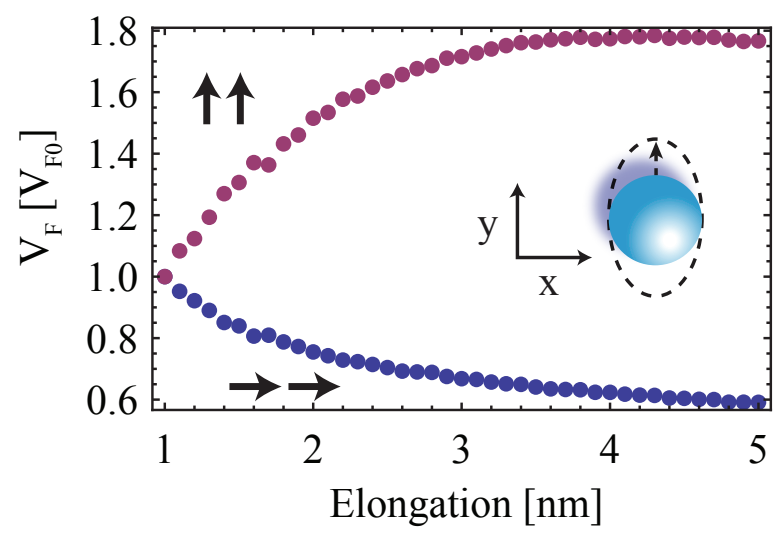

FIG. 2: Effect of the nanoparticle shape on the coupling between two nanoparticles. As shown in the inset, the shape of both particles (distance $5 \mathrm{~nm}$ ) is changed in the $y$ direction, starting from spherical particles with a radius of $1 \mathrm{~nm}$ and ending with an elongated shape; axis definition as in Fig. 1 (a). If the material dipoles are aligned with the $y$ axis, i.e. parallel to the axis of elongation, the coupling is enhanced starting from $\left|V_{F 0}\right|=0.168 \mathrm{meV}$. If the dipole alignment is in the $x$ axis, i.e. parallel to the separation vector, then the coupling is suppressed starting from $\left|V_{F 0}\right|=0.341 \mathrm{meV}$.

on the dielectric presence of additional nanoparticles. In the second part, we study electrical control of the Förster coupling with external electric fields in type I and type II quantum dots and elongated rod-shaped structures. If not otherwise mentioned, the material parameters are those of CdSe nanocrystals in a styrene matrix. The effective masses of the CdSe crystals are $m_{e l}^{*}=0.13 m_{0}$ for electrons and $m_{h}^{*}=0.45 m_{0}$ for holes, respectively. The relative dielectric constant of $\mathrm{CdSe}$ is $\epsilon_{r}=10.2$. The transition dipole moment defined by the Bloch wavefunction is chosen to be $|\mathbf{d}|=5.2 e \stackrel{\AA}{\text { [5] }}$. The static dielectric constant of a styrene matrix is $\epsilon_{r}=2.4$. We assume that the temperatures is sufficiently low such that the electron can be found in the lowest energy state of the conduction band and the hole can be found in the highest energy state of the valence band.

Results - Part I. In Fig. 1 (c), the Förster coupling is shown as a function of inter-particle separation for a twonanoparticle configuration. As depicted in the inset, the three setups for the two nanoparticles are sphere-sphere, spherespheroid, spheroid-spheroid. In the spherical cases, the particle diameter is taken to be $2 \mathrm{~nm}$, while in the non-spherical cases, the elongation (defined as the length of the semimajor axis of an ellipsoid) is taken to be $5 \mathrm{~nm}$. In this figure, the orientation of the material dipoles is in the same direction as the elongation; this orientation shows the most significant effects. For spherical nanoparticles, the dependence can be well fitted with a dipole-dipole $c / R^{3}$ law, where $c$ is the only fit parameter. Multipole terms of the envelope transition densities turn out not to be significant in this symmetric configuration. For the spheroid-shaped configurations, we obtain substantial deviations from a $c / R^{3}$ behavior at short distances. Multipole terms become significant and reduce the coupling. For long distances, the asymptotic behavior follows the usual dipole- 


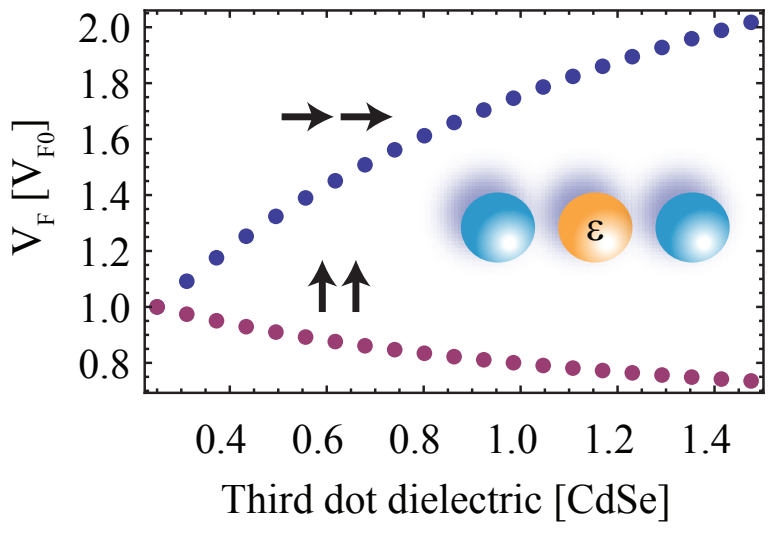

FIG. 3: Förster coupling as a function of the dielectric constant of a third dot in between. The setup is pictured in the inset (diameter of dots $2 \mathrm{~nm}$, distance $4.2 \mathrm{~nm}$ ). The coupling is either enhanced in the case of the material dipoles parallel to the separation vector (blue) or suppressed in the case of the material dipoles perpendicular to the separation vector (red). In the absence of the third dot the coupling is $\left|V_{F 0}\right|=0.58 \mathrm{meV}$ for the parallel case and $\left|V_{F 0}\right|=0.28 \mathrm{meV}$ for the perpendicular case.

\section{dipole law.}

In Fig. 2, the dependence of the Förster coupling on the shape of the nanoparticles is shown. The shape of the particles will affect the electron and hole wavefunctions and thus the transition density in the Förster matrix element. We elongate both particles along the $y$ direction, see Fig. 1 for the definition of the axes. We consider the two cases of parallel dipoles aligned along the $y$ axis and aligned along the $x$ axis. We observe an increase of the coupling for the $y$ axis alignment and a decrease of the coupling for the $x$ axis alignment. The substantial increase for the $y$ axis alignment (about $80 \%$ at an elongation of $5 \mathrm{~nm}$ ) can be rationalized by elongated wavefunctions in the $y$ direction with increasing elongation of the quantum dots. The derivative of the transition density is similar to a dipole that becomes larger and larger, which explains the increase in the coupling.

In Fig. 3, the Förster coupling of two quantum dots is shown as a function of the dielectric constant of a third dot in between the two. Depending on the orientation of the material dipole moment the coupling is either suppressed or increased. This can be intuitively explained by image dipoles due to the dielectric presence of the third dot. In the case of parallel dipoles perpendicular to the distance vector, the coupling is decreased because the two dots create opposite image dipoles within the third dot thereby suppressing the effective potential experienced by the other dot. In the case of parallel alignment of the dipole moments to the distance vector, the coupling increased by about $75 \%$ for a CdSe third dot. Here, the image dipoles are aligned parallel and effectively increase the potential created by one dot seen by the other. In Ref. [31], Hsu et $a l$. explain the behavior of the Förster coupling of two chromophores in a dielectric cavity along the same lines. A similar enhancement or suppression of the coupling depending on the molecular transition dipole orientation was found.
Results - Part II. To gain insight on the ability to actively engineer excitonic energy transfer, we now turn our attention to nanoparticles and excitons in the presence of electric fields. Metallic gates kept at a fixed potential modify the external electrostatic potential for the exciton, as indicated in Fig. 1 (b). We study the resulting effects on the Förster coupling of the nanoparticles. Note, however, that our combined solution of Schrödinger and Poisson equations can also be used to investigate the spectral overlap. Spatially varying electric fields could be designed such that different nanoparticles are tuned into resonance or energy gradients for directed exciton transfer are established. In the present work, the coupling of the excitons to surface plasmons of the metallic gates, as discussed and observed for example in [32,33], is neglected. This is justified because the time scale of exciton transport is of the order of tens of picoseconds while the time scale of the exciton-plasmon transfer is of the order of several nanoseconds [33].

In our present method, metallic gates are included by imposing arbitrarily complex boundary conditions in Poisson's equation. Intuitively, an electric field causes electron and hole in a single nanoparticle to spatially separate due to their different charge. Thus, the transition density, i.e. the electron-hole overlap, is decreased. As a consequence, the Förster matrix element between nanoparticles is reduced compared to the situation without an external field. This intuition is confirmed numerically on the real-space grid. Two quantum dots are sandwiched between infinite metallic gates, as pictured in the inset of Fig. 4 (a). Because of the geometry in this setup, the electric field is homogeneous throughout space and thus equal for both nanoparticles. The main figure shows the Förster coupling of the two nanoparticles as a function of the applied electric field. The dependence is almost Gaussian, as also predicted by a simplified model in [7]. The charge density plots of Fig. 4 (b) show indeed that in the presence of an electric field the electron and the hole separate in the quantum dot.

In the case of Fig. 4 (a), the radii of the dots are chosen to be $1 \mathrm{~nm}, 1.5 \mathrm{~nm}$ and $3 \mathrm{~nm}$ and their distances are $3 \mathrm{~nm}, 4.5 \mathrm{~nm}$, and $9 \mathrm{~nm}$, respectively. Note that for larger dots the electronhole separation occurs at smaller fields. This is explained by considering the length scale defined by the effective exciton Bohr radius. When this length scale is much larger than the size of the nanoparticle, the electron and hole wavefunctions are determined by the confinement potential. A large electric field is required for electron-hole separation. Conversely, in what may be called the bulk limit, when the nanoparticle is much larger than the exciton, the wavefunctions are determined by the Coulombic interactions. The electric field only has to be as large as to overcome the excitonic binding energy to separate electron and hole. As also borne out by our simulations, the relation of exciton size and nanoparticle size significantly affects the electric field dependence of the Förster coupling.

Up to this point, the nanoparticles in the simulations have been CdSe crystals. It is possible to engineer core-shell quantum dots $[34,35]$, for example of type II with a CdTe core and a CdSe shell or a CdSe core and a ZnTe shell [35]. We apply our method to such a type II quantum dot with a CdSe core 

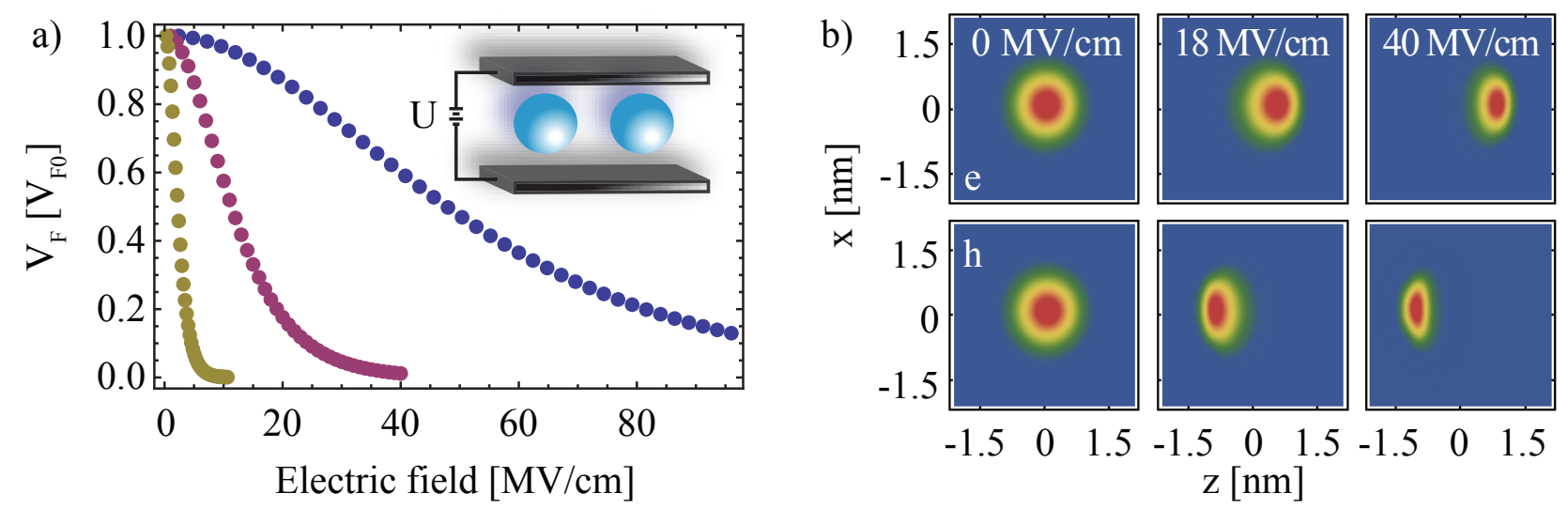

FIG. 4: (a) Förster coupling of two quantum dots as a function of an applied electric field. The dots are located between infinite metallic gates that are kept at a potential defined by the gate voltage $\mathrm{U}$. Three cases are considered: dot radius of $1 \mathrm{~nm}$ (blue), $1.5 \mathrm{~nm}$ (red), and $3 \mathrm{~nm}$ (yellow) and distances of $3 \mathrm{~nm}, 4.5 \mathrm{~nm}$, and $9 \mathrm{~nm}$, respectively. The Förster coupling shows a near-Gaussian dependence on the electric field for all dipole alignments, here depicted only for the alignment being parallel to the separation vector. The zero-voltage couplings for the three cases are $\left|V_{F 0}\right|=1.65 \mathrm{meV},\left|V_{F 0}\right|=0.49 \mathrm{meV}$, and $\left|V_{F 0}\right|=0.06 \mathrm{meV}$, respectively. (b) The electric field leads to a separation of electron charge density (upper panels) and hole charge density (lower panels) in the quantum dot.
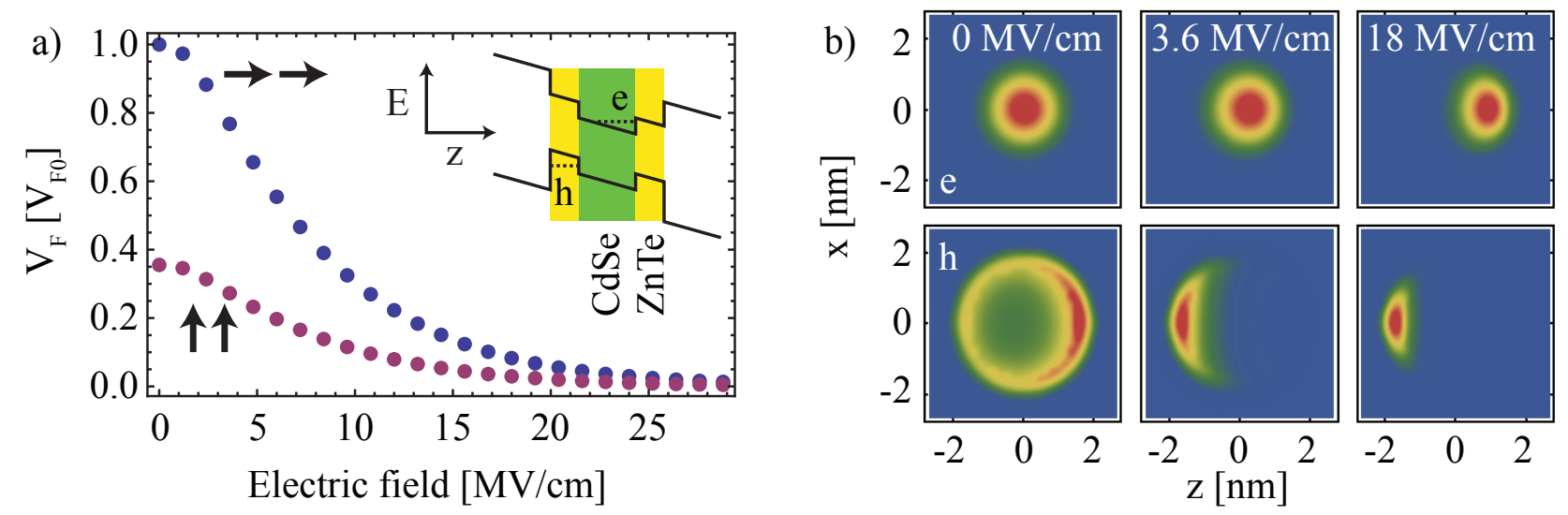

FIG. 5: Förster coupling between a type I and a type II quantum dot. (a) The inset shows the band structure of a type II quantum dot of CdSe with a ZnTe shell in the case of an applied electric field. The low-energy hole is localized in the shell, while the electron is in the core. The behavior of the Förster coupling as a function of the electric field is similar to the previous results, but overall smaller in magnitude and the electron-hole separation occurs at smaller electric fields (core radius $1.5 \mathrm{~nm}$, shell thickness $0.8 \mathrm{~nm}$, dot distance $4.5 \mathrm{~nm}$, and $\left|V_{F 0}\right|=0.39$ $\mathrm{meV}$ ). (b) The probability density of the electron (upper panels) and the hole (lower panels) as a function of applied electric field.

and a ZnTe shell $\left(m_{h}^{*}=0.2 m_{0}\right.$ and $\left.\epsilon_{r}=7.2\right)$. For this case, the bandstructure in depicted in the inset of Fig. 5 (a). The low-energy exciton transition density is formed by an electron mostly localized in the core and a hole mostly localized in the shell, see Fig. 5 (b) for a plot of the electron and hole charge densities. The Förster coupling is non-zero because the electron has a nonvanishing wavefunction amplitude in the shell and, vice versa, the hole has a nonvanishing amplitude in the core. Thus, the transition density is nonvanishing but the overall value of the coupling is smaller than for a type I quantum dot. When an electric field is applied electron and hole spatially separate in the dot and the transition density and hence the Förster coupling becomes smaller. The separation occurs at smaller electric fields than in the type I quantum dot case. As can be seen in the lower panels of Fig. 5 (b), the hole rapidly changes from being delocalized over shell and core at no electric field to being localized in the shell at one side of the quantum dot at small electric field.

In general, the layout of metallic gates is determined by the particular experiment, as an example consider the setup in Ref. [9]. In our final setup, see Fig. 6, we demonstrate the flexibility of our method in simulating arbitrarily complex geometries of gates and nanostructures. A quantum dot is placed next to an elongated, rod-shaped nanoparticle and metallic gates as depicted in the inset of Fig. 6. The metallic gates make it possible to control the electrostatic potential for the electron and the hole of the individual nanoparticles and thus the Förster coupling between them. The coupling shows a non-linear dependence when the voltage of the left gate is increased. Different physical effects compete: The electron and 


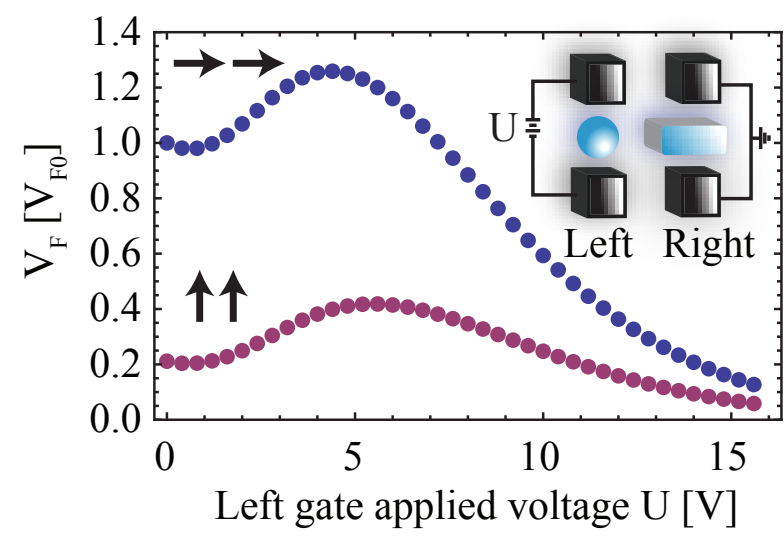

FIG. 6: Förster coupling in a complex dot-nanorod-gate setup (dot radius $4.5 \mathrm{~nm}$; nanorod radius $4.5 \mathrm{~nm}$ and $28 \mathrm{~nm}$ length; the gates are $50 \mathrm{~nm}$ apart). The coupling shows an optimal point as a function of the applied voltage on the left gate. In this case, $\left|V_{F 0}\right|=0.007$ $\mathrm{meV}$.

hole in the nanorod are attracted to their respective images in the left gate. Both electron and hole delocalize from the center of the nanorod to the left, thereby reducing the distance between the transition densities of dot and nanorod, which effectively increases the Förster coupling. At strong applied field, electron and hole separate in the dot, which eventually suppresses the coupling. Consequently, an optimal voltage in the intermediate regime is obtained.

\section{CONCLUSION}

We have studied the role of a complex dielectric environment and external electric fields in the Förster coupling of semiconductor nanoparticles. For this we have developed a technique, based on a self-consistent solution of the Schrödinger and Poisson equations, that allows us to incorporate excitonic binding energies, the shape dependence of the nanoparticles, a complex, spatially varying dielectric environment, and externally applied electric fields. We efficiently solve for the full Green's function of the general Poisson equation, including the boundary conditions for an arbitrarily complex electrostatic environment. This enables us to carry out theoretical predictions for nano-excitonic circuits, where the nature of the Förster interaction can be tuned by external potentials. We have simplified calculations by using the effective mass approximation and the Bloch-envelope decomposition. Atomistic calculations, although more accurate, at the moment cannot treat the macroscopic electrostatic environment. The main theoretical result is an intuitive expression for the Förster matrix element involving the gradient envelope transition densities of the nanoparticles that lends itself to extensions beyond the approximations employed in this work. Our results can be utilized to theoretically study and optimize energy transfer in novel engineered light-harvesting materials and excitonic circuits at the nano scale.

The authors thank A. Najmaie and S. Saikin for useful discussions. This material is based upon work supported as a part of the Center for Excitonics, as an Energy Frontier Research Center funded by the U.S. Department of Energy, Office of Science, Office of Basic Energy Sciences under Award number DE-SC0001088. We acknowledge the National Nanotechnology Infrastructure Network Computation project, NNIN/C, for computational resources.
[1] Scholes, G. D. Annu. Rev. Phys. Chem. 2003, 54, 57.

[2] May, V.; Kühn, V. Charge and Energy Transfer Dynamics in Molecular Systems, Wiley-VCH: Weinheim, 2004.

[3] Scholes, G. D.; Rumbles, G. Nat. Mat. 2006, 5, 683.

[4] Gur, I.; Fromer, N. A.; Geier, M.; Alivisatos, A. P. Science 2005, 310, 462.

[5] Crooker, S. A.; Hollingsworth, J. A.; Tretiak, S.; Klimov, V. I. Phys. Rev. Lett. 2002, 89, 186802.

[6] Lovett, B. W.; Reina, J. H.; Nazir, A.; Briggs, G. A. D. Phys. Rev. B 2003, 68, 205319.

[7] Nazir, A.; Lovett, B. W.; Barrett, S. D.; Reina, J. H.; Briggs, G. A. D. Phys. Rev. B 2005, 71, 045334.

[8] Becker, K.; Lupton, J. M.; Moller, J.; Rogach, A. L.; Talapin, D. V.; Weller, H.; Feldmann, J. Nat. Mater. 2006, 5, 777.

[9] High, A. A.; Novitskaya, E. E.; Butov, L. V.; Hanson, M.; Gossard, A. C. Science 2008, 321, 229.

[10] Förster, T. Ann. Physik 1948, 2, 55.

[11] Pons, T.; Medintz, I. L.; Sykora, M.; Mattoussi, H. Phys. Rev. B 2006, 73, 245302.

[12] Calarco, T.; Datta, A.; Fedichev, P.; Pazy, E.; Zoller, P. Phys. Rev. A 2003, 68, 012310 .
[13] Scholes G. D.; Andrews, D. L. Phys. Rev. B 2005, 72, 125331.

[14] Parascandolo G.; Savona, V. Phys. Rev. B 2005, 71, 045335.

[15] Allan G.; Delerue, C. Phys. Rev. B 2007, 75, 195311.

[16] Govorov, A. O. Phys. Rev. B 2005, 71, 155323.

[17] Degani M. H.; Hipolito, O. Phys. Rev. B 1987, 35, 9345.

[18] Altarelli, M.; Ekenberg, U.; Fasolino, A. Phys. Rev. B 1985 32, 5138.

[19] Efros, A. L.; Rosen, M.; Kuno, M.; Nirmal, M.; Norris, D. J.; Bawendi, M. Phys. Rev. B 1996 54, 4843.

[20] Klimeck G. et al., IEEE Transactions on Electron Devices 2007, 54, 2090; Korkusinski M. et al., Microelectronics Journal 2008, $39,318$.

[21] http://cms.mpi.univie.ac.at/vasp/

[22] Franceschetti A.; Zunger, A. Phys. Rev. Lett. 1997, 78, 915.

[23] Hu, J.; Wang, L.-W.; Li, L.-S.; Yang, W.; Alivisatos, A. P. J. Phys. Chem. B 2002, 106, 2447.

[24] McWeeny R. Methods of Molecular Quantum Mechanics, 2nd ed., Academic Press London, 1992; Krueger, B. P.; Scholes, G. D.; Fleming, G. R. J. Phys. Chem. B 1998, 102, 5378; Scholes, G. D.; Gould, I. R.; Cogdell, R. J.; Fleming, G. R. J. Phys. Chem. B 1999, 103, 2543; Tretiak, S.; Middleton, C.; Chernyak, 
V.; Mukamel S. J. Phys. Chem. B 2000, 104, 9540; Tretiak, S.; Middleton, C.; Chernyak, V.; Mukamel S. J. Phys. Chem. B 2000, 104, 4519.

[25] Schrier J.; Wang, L-W. J. Phys. Chem. C 2008, 112, 11158.

[26] Stopa, M. Phys. Rev. B 2001, 63, 195312.

[27] Sedlmaier, S.; Stopa, M.; Schedelbeck, G.; Wegscheider, W.; Abstreiter, G. Phys. Rev. B 2002, 65, 201304.

[28] Typically the valence band is p-like and has strong spin-orbit coupling (see Ref. [19]). Hole states have total angular momenta $J=3 / 2$ and $J=1 / 2$. For example, a state with $J=$ $3 / 2$ and $M_{J}=+3 / 2$ can be written: $u_{+3 / 2}=(X+i Y) \uparrow$ $/ \sqrt{2}$, where $X$ and $Y$ are $\mathrm{p}$ orbitals along the $\mathrm{x}$ and $\mathrm{y}$ crystal directions. The valence band degeneracy can be lifted, and different hole states mixed, by the crystal structure as well as by the nanoparticle boundary. Thus, for very small nanoparticles the excitonic electron dipole moment depends on the crystal shape.

[29] Manby F. R.; Knowles, P. J. Phys. Rev. Lett. 2001, 87, 163001.

[30] Bastard, G. Phys. Rev. B 1981, 24, 5693.

[31] Hsu, C.-P.; Fleming, G. R.; Head-Gordon, M. J. Chem. Phys. 2001, 114, 3065.

[32] Chang, D. E.; Sorensen, A. S.; Hemmer, P. R.; Lukin, M. D. Phys. Rev. Lett. 2006, 97, 053002.

[33] Akimov, A. V.; Mukherjee, A.; Yu, C. L.; Chang, D. E.; Zibrov, A. S.; Hemmer, P. R.; Park, H.; Lukin, M. D. Nature 2007, 450, 402.

[34] Steiner, D.; Dorfs, D.; Banin, U.; Della Sala, F.; Manna, L.; Millo, O. Nano Lett. 2008, 8, 2954.

[35] Kim, S.; Fisher, B.; Eisler, H.-J.; Bawendi, M. J. Am. Chem. Soc. 2003, 125, 11466. 